\title{
Data mining and biological sample exportation from South Africa: A new wave of bioexploitation under the guise of clinical care?
}

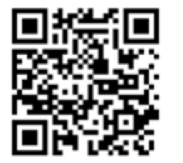

In September 2015, the South African (SA) health insurer Discovery announced that, in partnership with Craig Venter's company Human Longevity Inc., it would provide genetic testing to its members for USD250 (approximately ZAR3 400) per person. On the surface, this appears to be innovative and futuristic. However, a deeper look at this announcement reveals considerable problems in the exportation of biological samples and data out of SA, and brings into sharp focus the lack of protection in place for potential donors. In return for a reduced-cost genetic test, as part of that deal the deidentified information will be exported to and stored in Venter's company in the USA. ${ }^{[1]}$ It is possible that the data will be obtained from the samples in the USA, so the samples themselves will be exported. Genetic testing is being offered under the guise of clinical care, but for the apparent purpose of building up a large database for research in another country, and in so doing exposes the deficiencies of the SA regulatory framework on the use, storage and export of biological samples.

The data from these biological samples and the samples themselves are a valuable resource in medical research, helping to identify the roles that genes play in disease development and accelerating new drug development. For decades there has been a unidirectional flow of samples out of Africa to various destinations in developed countries, with no benefit to local populations or local researchers. Such 'parachute research' has impacted negatively on the development of local capacity, infrastructure and expertise. As genomic research is advancing in SA, every effort should be made to encourage its development and ensure that SA biological samples and data are used locally. International collaborations can further develop and improve local capacity, but this must be non-exploitative and involve a sharing of facilities, expertise and expense. A central feature of these collaborations often involves the sharing of samples and data among institutions within SA, across Africa and in the rest of the world. The H3Africa project, for example, seeks to make samples and data available among African collaborators for future research, necessitating the movement of samples across the H3Africa network. Central to this project is the importance of African leadership in the research, as well as strengthening control over the use of samples and data through its data and sample-sharing polices. ${ }^{[2,3]}$

However, this recent development by Discovery Health undermines the strengthening of African ownership of our samples and data, and is exactly the sort of exploitative behaviour that we must guard against. Although the relatively low cost of the genetic test offered by Discovery may be enticing, this exploits consumers and is reminiscent of safari research, as the data leave the country with no SA oversight or control, for the commercial benefit of a US company and Big Pharma. In a study involving 212 participants in SA, almost $40 \%$ were not supportive of researchers or other organisations financially benefiting from the use of their biological samples, and $43 \%$ expressed a desire to share some of the profits. ${ }^{[4]}$ However, the only benefit for Discovery members is the offering of a genetic test at a reduced rate that must come out of available day-to-day benefits. Discovery also promises access to genetic counselling, at a cost to be covered by the consumer. ${ }^{[5]}$ This additional benefit is enticing. However, there are only 30 genetic counsellors registered on the Health Professions Council of South Africa (HPCSA) register, of whom only 18 have a master's degree in genetic counselling. Discovery has indicated that genetic counselling will be offered by doctors, yet very few doctors have training in this field and fewer still can afford to spend time on genetic counselling and consent processes in their busy consultation rooms.

Exome testing by Discovery demonstrates the need for guidelines and clarity in the export of biological data. The SA export permit system for tissues currently offers limited protection for tissue donors. The export of biological samples out of SA is governed by the National Health Act 2003, specifically the Regulations Relating to the Import and Export of Human Tissue, Blood, Blood Products, 
Cultured Cells, Stem Cells, Embryos, Zygotes and Gametes 2012. ${ }^{[6]}$ A biological sample cannot be exported without a valid export permit, and only samples obtained under the terms of the Act can be exported. All applications must provide proof in writing that the biological sample will continue to be used within the terms of the Act once it is exported. The process requires that a register be established of all samples exported, thus ensuring that there is some oversight of the movement of samples out of SA. However, gaps in the regulations remain that must be addressed to protect the rights of participants as international collaborations are forged. Importantly, there is nothing in the regulations preventing Discovery from exporting the samples, unless an export permit is refused, and of concern is that there are no regulations overseeing the export of biological data from SA.

\section{Export of biological samples: \\ The consent concerns}

Under the National Health Act 2003, informed consent is required for participation in medical research. Participants must be informed of the purpose of the research and any risks and benefit. Consent can then be given based on this information. Section 55 similarly requires the informed consent of the participant prior to the removal of any tissue. Biological samples can be reused and are a potential valuable resource for future research. Alternative consenting arrangements such as broad, tiered and dynamic consent have therefore been proposed. The 2015 Department of Health guidelines explicitly incorporate broad and tiered consent and provide guidance for research ethics committees (RECs).

The Organisation for Economic Co-operation and Development $(\mathrm{OECD})$ guidelines on human biobanks require that all participants be informed of access to their sample, as well as transfer abroad. ${ }^{[7]}$ The guidelines recommend REC approval for all future research, but the SA health research regulations are silent on consent that is required prior to the export of biological samples. It must simply be demonstrated that the sample was obtained within the parameters of the National Health Act 2003, i.e. that consent was obtained prior to its removal, and that it is to be used within the terms of the Act. The H3Africa informed consent guidelines state that one of its goals is to make samples widely available to facilitate research. This will include transferring samples abroad to collaborators. Yet in a review of 1305 protocols submitted under the now repealed Human Tissues Act, it was found that fewer than $50 \%$ of the informed consent documents informed research participants of their intent to export samples. ${ }^{[8]}$ Sample donors do not appear to be aware of the intent or indeed the possibility of the removal of their samples from the country, and RECs do not have a formal oversight role to play. Although it appears that samples and data will be exported under Discovery's new scheme, the extent to which people who avail themselves of the test will be aware of the storage and use of their biological data is unclear.

Arguably broad consent could cover exportation of samples. Participants are consenting to the storage and reuse of their samples for future, unknown research, and this could cover research in another country. However, participants consent to the donation of their samples in accordance with the SA ethical and legal framework, and they can assume that the samples will be used in accordance with that framework. The right to withdraw samples is inherent in the process, but it may not be possible to exercise this right if samples are transferred abroad, as the sample will be used in accordance with the regulations of that country. Empirical evidence demonstrates that South Africans have clear preferences on the future use of their samples, including the exportation of the samples, and these preferences must be respected. ${ }^{[4]}$ The possibility of exportation must therefore be raised during the informed consent process.

Considering the importance of the sharing of samples in genomic biobank research, it is surprising that the revised 2015 Department of Health guidelines are silent on the export of samples. At the very least it would be expected that RECs should have some oversight function. Once a sample is exported, the SA health research regulations and RECs lose all oversight and jurisdiction over the sample. Any assurances that the participant may have received in respect of storage, future use and confidentiality can no longer be guaranteed, and the REC has no power to prevent its use in future studies that are contrary to those views. In any case, it has no way of knowing for what research the samples are to be used, and it in effect loses control of the sample once it leaves the country. Upon adoption of the broad consent model, the REC is tasked with the role of gatekeeper of the samples, so it should be satisfied that the sample will be used within the terms of the consent prior to its exportation. However, by not requiring its approval, the regulations appear to circumvent the oversight function of RECs, a role that is invested in them by the national ethics guidelines.

\section{The absence of a material transfer agreement (MTA)}

The transfer of samples raises important ownership and intellectual property (IP) rights issues flowing from the sample. To resolve these issues in advance, exchanges of biological samples are generally conducted under the auspices of an MTA. An MTA is a legally binding agreement that documents the rights of the provider and recipient of the sample and is generally seen as facilitating the sharing of samples while offering protections to local institutions. ${ }^{[9]}$ MTAs should describe the purpose of the transfer as well as any restrictions on the use of the sample. It may also stipulate the terms of any publication arising from the samples, ownership of the samples or new inventions from the samples, and any potential IP rights. ${ }^{[10]}$

Internationally, MTAs are a common feature of the transfer of samples endorsed by the OECD guidelines, the H3A High Level Principles on Ethics, Governance and Resource Sharing and the International Society for Biological and Environmental Repositories, among other organisations. ${ }^{[1]]}$ Considering the past exploitation of SA biological samples, it would be expected that an MTA be required under the SA regulatory system. However, this is surprisingly absent. The export permit system seems more concerned with tracking the movement of samples than protecting the rights of donors and research institutions in SA. The recently updated research ethics guidelines could have filled this vacuum and recommended the development of MTAs prior to transfer of samples. If not informed that their sample will be exported, donors will expect that their biological samples will be used in accordance with SA law. Health research regulations and guidelines differ across countries and may be lacking in some, so it cannot be guaranteed that the sample will be subject to the same protections.

Owing to the enforceability of the MTA, the provider of the sample can continue to exercise control over the sample, as stipulated in the MTA. Importantly, MTAs can also be used to enforce donor preferences, REC decisions and the protections of SA regulations on the use of the sample in other jurisdictions. It has also been suggested that RECs should have a role to play in ensuring that the terms of the MTA are in compliance with the local and national ethical guidelines. $^{[12]}$ The REC would need to approve the terms of the MTA and be satisfied about the future use of the sample, in light of the 
original consent. This would also address some of the deficiencies in the consent process.

Although an MTA is likely to be a requirement of transfer of biological samples in most research institutions in SA, the lack of national guidance on this point leaves institutions with the task of creating MTAs that may not consider the important role that MTAs can have in protecting the rights of the donors. MTAs are generally seen as necessary to protect the intellectual property following from the sample, but they can also be valuable tools in protecting donor rights.

\section{Exportation of data}

Genomic biobanking research not only stores large quantities of biological samples but also generates considerable data, and Discovery's announcement demonstrates the enormous commercial value of these data. Since the birth of the Human Genome Project, the focus has been on the release of data to promote the advancement of science through the Bermuda Principles of 1996 and the Fort Lauderdale Agreement of 2003. Sharing of both samples and data is now often a condition of funding. ${ }^{[13]}$ Yet the SA regulatory framework fails to address the complex issues involved in data sharing, illustrated by the Discovery development. It is primarily focused on consent, sample storage and sample reuse, requiring only that the confidentiality and privacy of donors are protected in the sharing of any data. Similar to the sharing of biological samples, the national ethics guidelines require REC approval prior to the sharing of data, but with no requirement to consult the REC in advance of exportation of the data, it is not clear whether this is taking place. The announcement from Discovery does not provide any indication of ethical approval or oversight, and this should be guarded against.

Unlike the samples themselves, data are not physically exported and would therefore not form part of the export permit system. However, it is possible for the sample to remain in SA but for the data arising from that sample to be exported, as may be in the case in Discovery's new initiative, necessitating the need for oversight on the access to data. Confidentiality and privacy of participants must be assured, and there should be oversight of data transfer. The Protection of Personal Information Act 2013 does strive to offer protection on the use of data, and it requires a binding contract (such as an MTA) and the consent of the donor prior to exportation. However, the Act only covers biometric data; this does include DNA, but it is not clear whether other types of data flowing from biological samples are covered.

The HPCSA has addressed this point, and its guidelines state:

'There must be justifiable reasons which should be provided to Research Ethics Committees for data and specimens to leave the country. This should only be done after a Material Transfer Agreement has been signed and submitted to the local Research Ethics Committee. ${ }^{\text {?[14] }}$

These guidelines pertain only to healthcare professionals in SA who are registered with the Council, and not to the wider scientific community. However, the protection of data is just as important as the samples themselves. REC oversight is necessary, and the requirement of an MTA or data transfer agreement should be a feature of the SA regulatory framework.

\section{Conclusion}

To protect the genomic heritage of the country, proper national oversight on the exportation of biological samples is necessary. Medical insurance companies should not be permitted to exploit SA genetic heritage, yet the existing export permit system provides little in the way of ethical oversight and is more concerned with keeping a register of the movement of samples than ensuring that the system protects the rights of participants. Other than the requirement that the donor consents to the donation of the sample, it appears that there is no requirement for donor consent or REC consent for export. The export permit system fails to consider the need for legal and ethical oversight of the preferences of donors on the use of either their sample or the rights arising out of the future use of the sample.

Public trust is crucial to the success of biobank research, and the removal of biological samples in the absence of any oversight as specified in the consent document may be an invasion of that trust. Equally, data should not be removed in this way. In an effort to have a public account of the movement of the samples, considerable gaps remain within the SA export permit system. Currently it offers no ethical oversight of the transfer of the samples and data, and the protection of local researchers is not required by law. International collaborative research raises specific ethical concerns, particularly when collaborators are from high-income countries. Such collaborations should be allowed to flourish and can be of considerable benefit to SA researchers, but they must not be at the expense of the local researcher, and our regulatory framework must protect the rights of both the donor and the researcher.

However, a revision of the 2012 regulations is not necessary to fill the vacuums identified here. Rather they could be addressed on a national code of practice governing the use, reuse and exportation of biological samples that requires ethical approval and an MTA prior to the removal of samples, and that exportation of samples should be raised during the informed consent process. In the interim, MTAs can fill the gap left by the regulations and stipulate that the sample and data are used in accordance with national ethics guidelines and the terms of the original consent.

\section{Staunton, K Moodley}

Centre for Medical Ethics and Law, Faculty of Medicine and Health Sciences, Stellenbosch University, Tygerberg, Cape Town, South Africa

\section{Corresponding author: C Staunton (ciarastaunton@sun.ac.za)}

1. Cassidy S. Discovery to offer genetic testing. http://www.iol.co.za/lifestyle/discovery-to-offer-genetic testing-1.1920337\#.VhJuuCvd2y5 (accessed 20 October 2015).

2. H3Africa High Level Principles on Ethics, Governance and Resource Sharing. http://h3africa.org/ H3Africa High Level Principles on Ethics, Governance
about/ethics-and-governance (accessed 6 January 2016).

Ramsay M. Growing genomic research on the African continent: The H3Africa Consortium. S Afr Med J 2015;105(12):1016-1017. [http://dx.doi.org/10.7196/SAMJ.2015.v105i12.10281]

4. Moodley K, Sibanda N, February K, Rossouw T. 'It's my blood': Ethical complexities in the use, storage and export of biological samples: Perspectives from South African research participants. BMC Med Ethics 2014;15:4. [http://dx.doi.org/10.1186/1472-6939-15-4]

5. Discovery. Product enhancement in 2016. https://www.discovery.co.za/portal/individual/medical-aidnews-product-enhancements-2016 (accessed 6 January 2016).

6. http://www.sashg.org/documents/GovGazette2Mar2012.pdf (accessed 6 January 2016).

7. OECD Guidelines on Human Biobanks and Genetic Research Databases 2009. http://www.oecd.org/ sti/biotech/44054609.pdf (accessed 20 October 2015).

8. Sathar A, Dhai A, van der Linder S. Collaborative international research: Ethical and regulatory issues pertaining to human biological materials at a South African institutional research ethics committee. Dev World Bioeth 2014;14(3):150-157. [http://dx.doi.org/10.1111/dewb.12018]

9. Ramsay MJ, de Vries J, Soodyall H, Norris S, Sankoh O. Ethical issues in genomic research on the Ramsay MJ, de Vries J, Soodyall $\mathrm{H}$, Norris $\mathrm{S}$, Sankoh $\mathrm{O}$. Ethical issues in genomic research on the
African continent: Experiences and challenges to ethics review committees Human Genomics African continent: Experiences and challenges to ethics

10. Rodriguez V. Material transfer agreements: Open science vs. proprietary claims. Nat Biotechnol 2005;23(4):489-491. [http://dx.doi.org/10.1038/nbt0405-489]

11. International Society for Biological and Environmental Repositories. Best Practices for Repositories: Collection, Storage, Retrieval and Distribution of Biological Materials for Research. 3rd ed., 2011. Biopreserv Biobank 2012;10(2):81-161.

12. Chalmers D, Nicol D, Nicolas P, Zeps N. A role for research ethics committees in exchanges of human biospecimens through material transfer agreements. J Bioeth Inq 2014;11(3):301-306. [http://dx.doi. org/10.1007/s11673-014-9552-1]

13. Knoppers BM, Harris J, Tasse AM, et al. Towards a data sharing code of conduct for international genomic research. Genome Med 2011;3:46. [http://dx.doi.org/10.1186/gm262]

14. Health Professions Council of South Africa. Ethical Guidelines for Good Practice in the Health Care Professions: General Ethical Guidelines for Health Researchers. Book 6. http://www.hpcsa.co.za/ Professions: General Ethical Guidelines for Health Researchers. Book 6. http://www.hpcsa.co.za/
Uploads/editor/UserFiles/downloads/conduct_ethics/rules/generic_ethical_rules/booklet_6_gen_ ethical_guidelines_for_researchers.pdf (accessed 17 October 2014). 